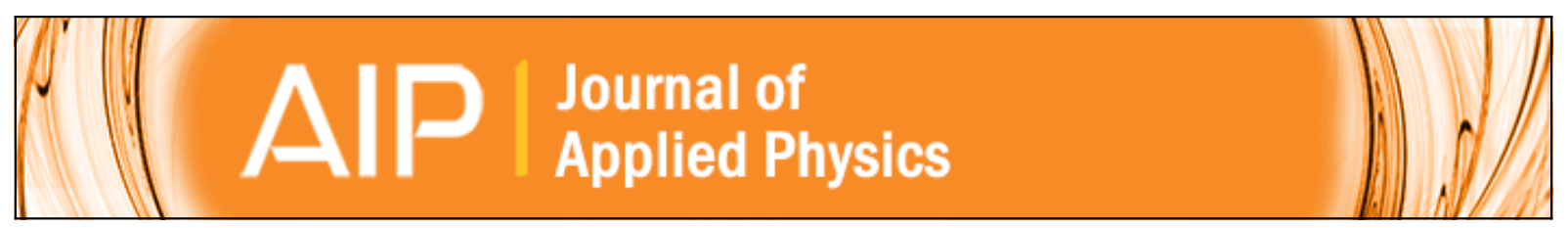

\title{
Maximizing the amplitude of coherent phonons with shaped laser pulses
}

Toru Shimada, Christian Frischkorn, Martin Wolf, and Tobias Kampfrath

Citation: Journal of Applied Physics 112, 113103 (2012); doi: 10.1063/1.4767922

View online: http://dx.doi.org/10.1063/1.4767922

View Table of Contents: http://scitation.aip.org/content/aip/journal/jap/112/11 ?ver=pdfcov

Published by the AIP Publishing

\section{Articles you may be interested in}

Optimal shaping of acoustic resonators for the generation of high-amplitude standing waves

J. Acoust. Soc. Am. 136, 1003 (2014); 10.1121/1.4892751

Coherent acoustic phonon generation and detection by femtosecond laser pulses in ZnTe single crystals

J. Appl. Phys. 114, 093513 (2013); 10.1063/1.4820518

Photoinduced multimode coherent acoustic phonons of metallic nanoprisms and the effects of shape-induced anisotropic electronic stresses

J. Chem. Phys. 134, 184506 (2011); 10.1063/1.3590373

Coupling of ultrafast laser energy to coherent phonons in bismuth

Appl. Phys. Lett. 90, 251111 (2007); 10.1063/1.2750401

Effect of intense chirped pulses on the coherent phonon generation in $\mathrm{Te}$

Appl. Phys. Lett. 90, 071901 (2007); 10.1063/1.2476306

A|P| $\left.\right|_{\text {Letters }} ^{\text {Applied Physics }}$

is pleased to announce Reuben Collins as its new Editor-in-Chief

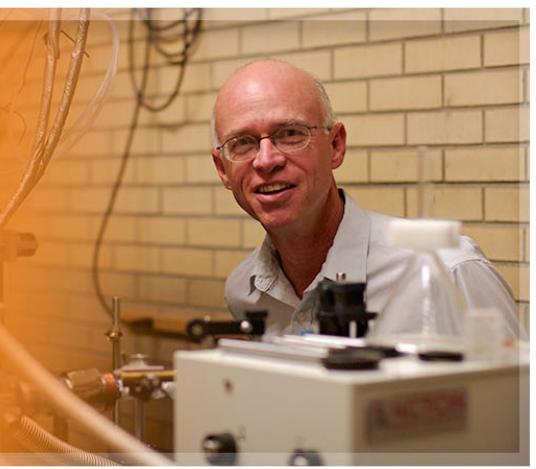




\title{
Maximizing the amplitude of coherent phonons with shaped laser pulses
}

\author{
Toru Shimada, ${ }^{1, a)}$ Christian Frischkorn, ${ }^{2}$ Martin Wolf, ${ }^{1}$ and Tobias Kampfrath ${ }^{1}$ \\ ${ }^{1}$ Fritz-Haber-Institut der Max-Planck-Gesellschaft, Faradayweg 4-6, 14195 Berlin, Germany \\ ${ }^{2}$ Fachbereich Physik, Freie Universität Berlin, Arnimallee 14, 14195 Berlin, Germany
}

(Received 24 October 2012; accepted 31 October 2012; published online 5 December 2012)

\begin{abstract}
We perform model calculations of coherent lattice vibrations in solids driven by ultrashort laser pulses. In order to maximize the amplitude of the coherent phonon in the time domain, an evolutionary algorithm optimizes the driving laser field. We find that only a Fourier-limited single pulse yields the maximum phonon amplitude, irrespective of the actual physical excitation mechanism (impulsive or displacive). This result is in clear contrast to the widespread intuition that excitation by a pulse train in phase with the oscillation leads to the largest amplitude of an oscillator. We rationalize this result by an intuitive model and discuss implications for other nonlinear processes such as optical rectification. () 2012 American Institute of Physics. [http://dx.doi.org/10.1063/1.4767922]
\end{abstract}

\section{INTRODUCTION}

It is a longstanding dream of laser spectroscopy to control chemical transformations of molecules by light. One approach to this goal are tailored laser pulses that steer the motion of nuclei along a selected path. ${ }^{1}$ In solids, femtosecond laser pulses are routinely used to launch vibrational wavepackets, so-called coherent phonons, resulting in a classical oscillation of the crystal lattice. ${ }^{2}$ A very exciting prospective is to induce very large oscillation amplitudes, which would allow one to study lattice vibrations beyond the harmonic regime such that different lattice modes start to interact with each other. Exciting phenomena along these lines are atomic displacements with nonzero average $\mathrm{e}^{3}$ or the induction of localized lattice deformations. ${ }^{4}$ An ultimate goal would be the ultrafast triggering of structural phase transitions. ${ }^{5}$

Therefore, one needs to find the optimum shape of the driving laser pulse that generates a maximum lattice amplitude $Q(t)$ at a certain instant of time. Previous works ${ }^{6-10}$ made use of pulse shaping techniques and indicated that dividing a pulse into a pulse train should lead to an increased phonon amplitude, provided the pulse repetition rate equals the phonon frequency. This process can be compared to kicking a pendulum with appropriate timing. However, recently, Misochko et al. suggested that a pulse train does not enhance the phonon amplitude as compared to single-pulse excitation, provided the total optical energy is the same in both scenarios. ${ }^{11}$ Thus, it is not yet clear which shape of the laser pulse excites coherent phonons most efficiently, in terms of realizing the largest oscillation amplitude.

In this paper, we address the question of the optimum laser pulse to excite coherent phonons more generally. We perform simulations of the coherent-phonon dynamics with an evolutionary algorithm to find the pulse shape that maximizes the amplitude of the lattice vibration in the time domain. We find that for a given laser intensity spectrum, the Fourier-limited single pulse with identical intensity spectrum yields the best result. Simulations are performed for two dif-

\footnotetext{
a) Present address: Hirosaki University, 1 Bunkyo-cho, Hirosaki, Aomori 036-8152, Japan. Electronic mail: tshimada@cc.hirosaki-u.ac.jp.
}

ferent types of excitation mechanisms, but general considerations show that our numerical results are valid for all types of excitation processes that scale linearly with the laser intensity profile.

\section{MODEL AND IMPLEMENTATION}

Figure 1 shows the model schematic of our study. A Fourier-limited pulse with electric field $E_{\mathrm{FL}}(t)$ is transformed into a pulse

$$
E(t)=\left(H * E_{\mathrm{FL}}\right)(t)=\int \mathrm{d} t^{\prime} H\left(t-t^{\prime}\right) E_{\mathrm{FL}}\left(t^{\prime}\right),
$$

by means of a pulse shaper with a freely programmable transfer function $H(t)$, where $t$ denotes time. The laser field $E(t)$ then creates a time-dependent force $F(t)$ onto a vibrational mode characterized by the coordinate $Q(t)$. This force is a result of inelastic light scattering, and its temporal structure depends on the shape of the laser pulse as well as of the properties of the solid. ${ }^{12}$ In case of transparent materials, impulsive stimulated Raman scattering (ISRS) is operative, and $F(t)$ is proportional to the instantaneous laser intensity, ${ }^{13} F(t) \propto E(t)^{2}$. In opaque materials, another mechanism, displacive excitation of coherent phonons (DECP), usually dominates. Then, the generation of electron-hole pairs induces a force $F(t)$ that scales with the absorbed laser energy, ${ }^{14,15} F(t) \propto \int_{-\infty}^{t} \mathrm{~d} t^{\prime} E\left(t^{\prime}\right)^{2}$. One can summarize these cases in the following equation:

$$
F(t)=\left(C_{F E} * E^{2}\right)(t),
$$

where $C_{F E}(t)$ describes the coupling of the light field $E$ with the lattice. Its temporal shape represents the force $F$ acting on the lattice as a result of a $\delta$-like laser pulse. $C_{F E}(t)$ equals the $\delta$-function and the Heaviside step function in case of ISRS and DECP, respectively. For the sake of simplicity, we assume that $F(t)$ drives only one phonon mode that can be represented by a harmonic oscillator with resonance frequency $\omega_{0}$ and damping constant $\gamma^{12}$ Thus, the impulse response of this mode is given by the Green's function

$$
C_{Q F}(t)=\exp (-\gamma t) \sin \left(\omega_{0} t\right),
$$




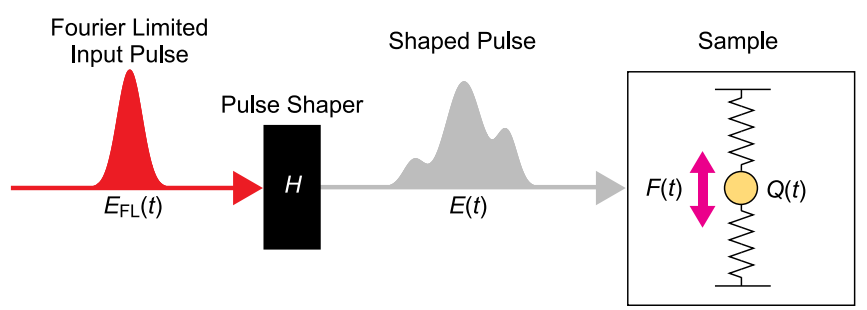

FIG. 1. Model schematic of our study. A Fourier-limited input pulse $E_{\mathrm{FL}}$ is shaped by a freely programmable pulse shaper with filter function $H$. The resulting pulse $E(t)$ exerts a force $F(t)$ onto a phonon mode, inducing a timedependent displacement $Q(t)$. The goal is to reach a maximum amplitude in the time-domain.

and the dynamics of the phonon amplitude as a result of the force $F(t)$ follows from the convolution

$$
Q(t)=\left(C_{Q F} * F\right)(t)=\left[C_{Q F} * C_{F E} *\left(H * E_{\mathrm{FL}}\right)^{2}\right](t) .
$$

We aim to find the best filter function $H$ that maximizes the peak amplitude $\max _{t}|Q(t)|$. This task is highly nontrivial as $H$ shows up in a nonlinear manner in Eq. (4). Note that there are two boundary conditions. First, the pulse shaper is a passive device such that $|\tilde{H}(\omega)| \leq 1$ where the tilde denotes the Fourier transform and $\omega$ denotes the angular frequency. Second, the pulse shaper has to fulfill causality, that is, $H(t)=0$ if $t<0$. In our simulations, however, we do not apply this constraint as the optimum $H$ is found to fulfill causality anyway.

For the numerical implementation of this optimization problem, we apply a genetic algorithm ${ }^{16}$ to amplitude $A$ and phase $\varphi$ of the filter function

$$
\tilde{H}(\omega)=A(\omega) \mathrm{e}^{\mathrm{i} \varphi(\omega)},
$$

in the frequency domain. The intensity envelope of the initial, Fourier-limited laser pulse (duration $30 \mathrm{fs}$ ) is shown in
Fig. 2(a), and the corresponding intensity spectrum is plotted in Fig. 2(b). In order to fully cover the laser spectrum $\left|\tilde{E}_{\mathrm{FL}}(\omega)\right|^{2}$, we choose the wavelength range from $2 \pi c / \omega$ $=730 \mathrm{~nm}$ to $860 \mathrm{~nm}$. This window is represented by an equidistant frequency mesh with a resolution of $\Delta \omega / 2 \pi$ $=1 / 16.1 \mathrm{ps}$, which corresponds to a time span of more than three lifetimes $1 / \gamma$ of the phonon mode chosen (frequency $\left.\omega_{0} / 2 \pi=10 \mathrm{THz}, 1 / \gamma=5 \mathrm{ps}\right)$.

We start the optimization with a set $\{(A, \varphi)\}$ of 20 randomly generated phase and amplitude functions (genes) that constitute the first generation. For each gene, we calculate the phonon dynamics $Q(t)$ using $H$ and Eq. (4). The maximum phonon amplitude

$$
f=\max _{t}|Q(t)|,
$$

in the time domain serves as the fitness function that has to be maximized. The next generation of genes is obtained by adopting the two genes with the highest fitness (elites) whereas the remaining 18 genes are generated by crossover and/or mutation operations of the old genes. With iteration, the fitness of the elites is successively improved and is believed to finally converge to the global maximum. ${ }^{17}$ It is convenient to keep the temporal center of mass of the pulse at time zero, which is achieved by adding an appropriate term linear in $\omega$ to the spectral phase $\varphi$ of each gene.

\section{RESULTS}

Figure 2(c) shows the progress of the optimization, both for the case of ISRS- and DECP-type excitation. The fitness $f$ is normalized to that of a Fourier-limited pulse $(\tilde{H}=1)$. It starts at $\approx 0.05$ and then increases monotonously. Note that $f$ saturates after about 15000 generations at a value of 1.0, which equals the fitness of a Fourier-limited pulse. In order to understand the evolution of the optimization process in
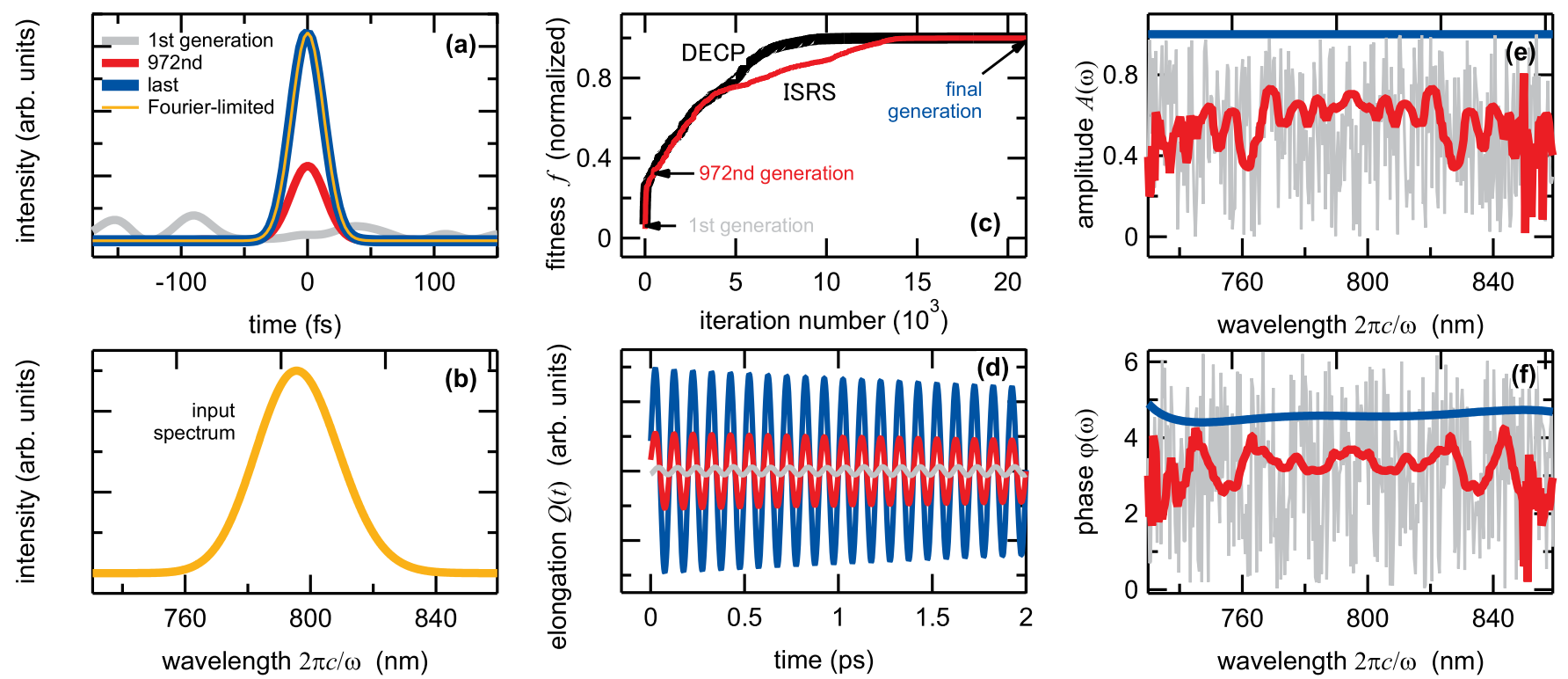

FIG. 2. Optimizing laser-driven lattice displacement. (a) Intensity profiles $I(t)$ of the Fourier-limited laser pulse and the fittest, shaped pulses from the first, 972nd, and last generation of the optimization process. (b) Intensity spectrum of the Fourier-limited pulse. (c) Progress of the optimization process for the case of ISRS and DECP excitation of the coherent phonon mode at $\omega_{0} / 2 \pi=10 \mathrm{THz}$. The fitness $\max _{t}|Q(t)|$ is normalized to that of the Fourier-limited input pulse. Arrows mark the selected generations shown in (a), (d)-(f) for the case of ISRS-type excitation. (d) Simulated coherent phonon dynamics for pulses shown in (a). (e) Spectral amplitude $A$ and (f) phase $\varphi$ of the pulse shaper's filter function for the pulses shown in (a). 
more detail, we inspect the shape of the driving laser pulse, the filter function, and the resulting phonon dynamics of the fittest gene of the first generation (corresponding to a completely random $\tilde{H}$ ), as well as those of a later generation (exemplarily, the 972nd is chosen) and of the last iteration [see arrows in Fig. 2(c)].

As illustrated in Fig. 2(d), the phonon dynamics $Q(t)$ displays a damped harmonic oscillation and shows how the peak displacement increases with increasing generation number. The three associated laser pulses are shown in Fig. 2(a). While the envelope of the pulse intensity is distributed over the whole simulated time window of $16.1 \mathrm{ps}$ for the first generation, it is already more concentrated at time zero at the 972nd generation. At the last generation, the laser pulse has turned into a pulse that cannot be distinguished from the Fourier-limited pulse any more. Finally, Figs. 2(e) and 2(f) show the evolution of the pulse shaper's filter function $\tilde{H}(\omega)$. It starts with a function whose spectral amplitude and phase fluctuate randomly over the full parameter range from 0 to 1 and 0 to $2 \pi$, respectively. In the course of the optimization, however, $A(\omega)$ and $\varphi(\omega)$ become flatter and flatter, with the phase approaching a constant and the amplitude converging to 1 . Therefore, the final optimized pulse is indeed Fourier-limited.

We note that repeated runs of the genetic algorithm are found to deliver virtually the same results, apart from an offset of the spectral phase that varies from run to run. The slight variations of $\varphi$ at the edges of the frequency window are not relevant as the spectral intensity of the laser pulse [Fig. 2(b)] is already very small there.

\section{DISCUSSION}

How can one understand that a Fourier-limited pulse leads to the largest phonon amplitude? To answer this question, we consider Eq. (4) showing that the displacement $Q(t)$ is the response $C_{Q F} * C_{F E}$ to a $\delta$-pulse, yet smeared out by convolution with the intensity envelope of the actual laser pulse. This scenario is illustrated in Fig. 3 for the case of ISRS $\left[C_{F E}(t) \propto \delta(t)\right]$ : The driving laser pulse can be understood as a superposition of appropriately timed and scaled $\delta$-pulses each of which triggers an exponentially damped lattice vibration [Eq. (3)]. The superposition (addition) of all these sub-responses finally yields the total lattice displacement $Q(t)$. Figure 3 makes clear that maximum amplitude is achieved only when the laser-pulse envelope overlaps with the maximum of $\left|C_{O F} * C_{F E}\right|$ as tightly as possible. This condition is fulfilled when $E(t)^{2}$ is as short as possible. As for a given intensity spectrum, a Fourier-limited pulse has the shortest pulse duration of all possible waveforms, this pulse will yield the maximum phonon amplitude, in agreement with our simulations. Note that the Fourier-limited pulse has to be shorter than the width of the maximum of $\left|C_{Q F} * C_{F E}\right|$, which is given by half a vibrational period $\pi / \omega_{0}$ in both cases ISRS and DECP.

We finally compare our results to relevant experimental work. First, the authors of Ref. 11 used a single pulse and a double pulse with the same total energy to generate coherent phonons in Te. They found that the double excitation did not

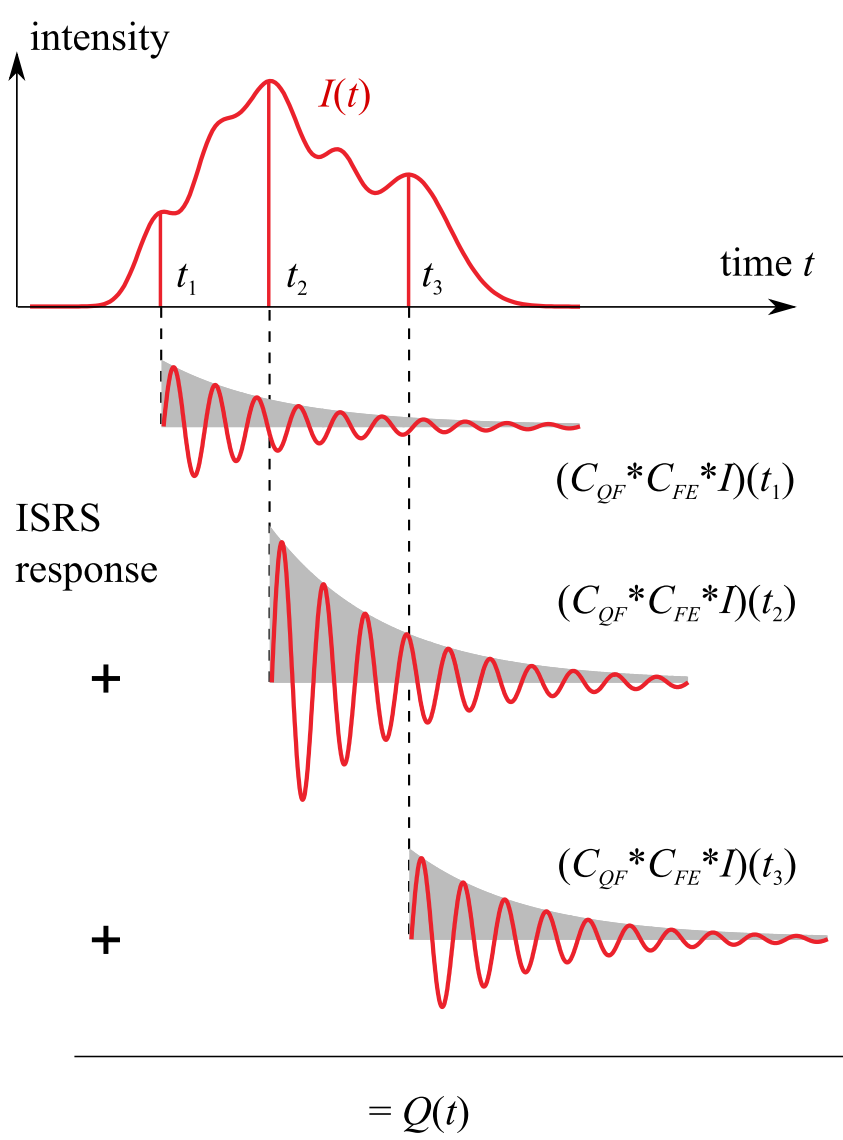

FIG. 3. Illustration of Eq. (4) describing the phonon excitation process for the case of ISRS-type coupling. Coherent-phonon excitation may be understood as a sequential excitation by a series of $\delta$-like pulses that follow the intensity envelope $I(t)$ of the excitation pulse (topmost curve). The resulting displacement $Q(t)$ is a sum of all these events, three of which are indicated in the figure.

lead to an increased vibrational amplitude, consistent with our findings. Second, in Ref. 18, the linear chirp of the pump pulse was varied over a wide parameter range, and a maximum amplitude of the resulting coherent lattice vibrations in Te was found for a Fourier-limited pulse, in full agreement with our work. It should be mentioned that in Ref. 18, pulses with positive and negative chirp yet same magnitude and therefore identical intensity envelope yielded different phonon amplitudes. This behavior is beyond the validity range of our model in which the intensity envelope of the pump pulse fully determines the induced phonon dynamics [see Eqs. (2) and (4)]. To remedy this discrepancy, our model would have to be extended, possibly by a force formula [Eq. (2)] that takes more details of the driving laser field $E(t)$ into account, for instance, its instantaneous frequency. To account for more intense pump pulses, nonlinearities higher than second order with respect to $E(t)$ (such as multiphoton absorption ${ }^{18}$ and time-dependent phonon frequencies due to vibrational softening/hardening by excited electrons) as well as anharmonicities of the phonon dynamics should be included. Note, however, that all these aspects are beyond the scope of this work. We finally would like to emphasize again that even under the more complex excitation conditions of Ref. 18, the optimum pump pulse had zero chirp, consistent with our results. 


\section{CONCLUSION}

In conclusion, we have shown that, for a given intensity spectrum, Fourier-limited laser pulses yield the largest amplitude of laser-induced coherent lattice vibrations. We note that these findings are relevant also to other second-order effects with respect to the driving laser field. An important example is optical rectification of femtosecond laser pulses in nonlinear optical crystals, a process which is routinely used to generate terahertz radiation. ${ }^{19}$ Consequently, secondorder mixing processes provide the highest terahertz field amplitudes when Fourier-limited generation pulses are used.

T.S. gratefully acknowledges supports by the Alexander von Humboldt Stiftung and the Seki memorial foundation for science.

${ }^{1}$ D. J. Tannor, R. Kosloff, and S. A. Rice, J. Chem. Phys. 85, 5805 (1986).

${ }^{2}$ T. Dekorsy, G. C. Cho, and H. Kurz, in Topics in Applied Physics: Light Scattering in Solids VIII edited by M. Cardona and G. Güntherrodt (Springer, 2000), Vol. 76, p. 169.

${ }^{3}$ M. Först, C. Manzoni, S. Kaiser, Y. Tomioka, Y. Tokura, R. Merlin, and A. Cavalleri, Nat. Phys. 7, 854 (2011).

${ }^{4}$ A. J. Sievers and S. Takeno, Phys. Rev. Lett. 61, 970-973 (1988).

${ }^{5}$ S. Wall, D. Wegkamp, L. Foglia, K. Appavoo, J. Nag, R. F. Haglund, J. Stähler, and M. Wolf, Nat. Commun. 3, 721 (2012).
${ }^{6}$ A. M. Weiner, D. E. Leaird, G. P. Wiederrecht, and K. A. Nelson, Science 247, 1317 (1990).

${ }^{7}$ T. Dekorsy, W. Kütt, T. Pfeifer, and H. Kurz, Europhys. Lett. 23, 223 (1993).

${ }^{8}$ M. Hase, K. Mizoguchi, H. Harima, S. Nakashima, M. Tani, K. Sakai, and M. Hangyo, Appl. Phys. Lett. 69, 2474 (1996).

${ }^{9}$ A. Rundquist, A. Efimov, and D. H. Reitze, J. Opt. Soc. Am. B 19, 2468 (2002).

${ }^{10}$ C. A. D. Roeser, M. Kandyla, A. Mendioroz, and E. Mazur, Phys. Rev. B 70, 212302 (2004).

${ }^{11}$ O. V. Misochko, M. V. Lebedev, H. Schäfer, and T. Dekorsy, J. Phys.: Condens. Matter 19, 406220 (2007).

${ }^{12}$ R. Merlin, Solid State Commun. 102, 207 (1997).

${ }^{13}$ T.-X. Yan, E. B. Gamble, Jr., and K. A. Nelson, J. Chem. Phys. 83, 5391 (1985).

${ }^{14}$ H. J. Zeiger, J. Vidal, T. K. Cheng, E. P. Ippen, G. Dresselhaus, and M. S. Dresselhaus, Phys. Rev. B 45, 768 (1992).

${ }^{15}$ H. L. Fragnito, J.-Y. Bigot, P. C. Becker, and C. V. Shank, Chem. Phys. Lett. 160, 101 (1989).

${ }^{16}$ The genetic algorithm software is included in the spatial light modulator (SLM-S640d/320d) produced by JENOPTIK Optical Systems GmbH, Germany.

${ }^{17}$ E. Schöneburg, F. Heinzmann, and S. Feddersen, Genetische Algorithmen und Evolutionsstrategien (Addison-Wesley, New York, 1994).

${ }^{18}$ O. V. Misochko, T. Dekorsy, S. V. Andreev, V. O. Kompanets, Yu. A. Matveets, A. G. Stepanov, and S. V. Chekalin, Appl. Phys. Lett. 90, 071901 (2007).

${ }^{19}$ K.-L. Yeh, M. C. Hoffmann, J. Hebling, and K. A. Nelson, Appl. Phys. Lett. 90, 171121 (2007). 